\title{
Automaticity without extensive training: The role of memory retrieval in implementation of task-defined rules
}

\author{
Motonori Yamaguchi • Robert W. Proctor
}

Published online: 2 February 2011

(C) Psychonomic Society, Inc. 2011

\begin{abstract}
Although the concept of automaticity is closely associated with extensive rote training, previous studies have shown that task-defined stimulus-response ( $\mathrm{S}-\mathrm{R}$ ) mappings can be implemented in parallel and involuntarily, without much training, as if they are automatically processed. An irrelevant task context may trigger a task-defined rule because the rule is actively maintained in working memory, resulting in erroneous implementation of that rule. However, the present study demonstrated that active maintenance of task rules is not necessary for their automatic implementation. Instead, the results are consistent with the memory view of automaticity, according to which task-defined S-R rules are implemented via automatic retrieval of S-R episodes.
\end{abstract}

Keywords Executive control · Automaticity · Instance theory · Task switching $\cdot$ Working memory $\cdot$ Response congruity

Although it is typically assumed that automaticity can be attained only after repeatedly performing the same task for a long period of time within an invariant task context (see, e.g., Schneider \& Shiffrin, 1977), there is evidence that certain cognitive tasks can be performed in an automatic fashion without extensive training (Logan \& Etherton, 1994). Such findings are consistent with the memory view of automaticity (Logan, 1988), according to which automatic processes are

M. Yamaguchi $\cdot$ R. W. Proctor

Department of Psychological Sciences, Purdue University,

Purdue, IN, USA

M. Yamaguchi $(\square)$

Department of Psychology, Vanderbilt University,

Nashville, TN 37203, USA

e-mail: motonori.yamaguchi@vanderbilt.edu those based on retrieval of episodic memory about past events. It states that an automatic process can occur as long as there is an instance of a past event stored in long-term memory (LTM), which may be established by a single encounter with a stimulus-response (S-R) event (Waszak, Hommel, \& Allport, 2003) or by merely memorizing S-R associations (Logan \& Klapp, 1991; Zbrodoff, 1999). Consequently, the memory view predicts that task-defined S-R mappings can be implemented automatically without extensive task training.

Evidence supporting this prediction comes from studies that show response-congruity effects. In a dual-task study, for instance, Hommel (1998) showed that the response time (RT) for Stimulus 1 (S1) is shorter when Response 1 (R1) is compatible with Response 2 (R2). The influence of R2 on $\mathrm{R} 1$ implies that $\mathrm{R} 2$ selection can proceed in parallel with R1 selection, as if task-defined S-R translation is an automatic process, challenging the traditional "bottleneck" account of dual-task interference (Pashler, 1994). Similarly, without extensive training, task-irrelevant noise stimuli (flankers) can exert influences on response selection (Eriksen \& Eriksen, 1974), in the way that RTs are shorter when responses mapped to the target and flankers are congruent. A recent study (Cohen-Kdoshay \& Meiran, 2007) suggested that this flanker effect can occur on the very first trial, implying that instructions are sufficient for the effect to occur.

Given that little or no training is required, the responsecongruity effect seems to support the memory view of automaticity (e.g., Altmann \& Gray, 2008; Schneider \& Logan, 2009). However, there is another account that explains these phenomena without assuming memory retrieval. According to the active-maintenance account, automatic implementation of task-defined mappings occurs because the task requires these mappings to be maintained actively in working memory (WM; see, e.g., Meiran, 1996; 
Rogers \& Monsell, 1995). Because both a dual task and the flanker task require multiple S-R mappings to be kept active simultaneously in WM, encoding of irrelevant stimuli may automatically trigger implementation of erroneous mapping rules and activate the responses associated with them. Hence, in the active-maintenance account, memory retrieval does not contribute to the automaticity of task-defined rules.

To distinguish the two accounts of automatic S-R translation, one can assess whether the response-congruity effect occurs when irrelevant S-R mappings are unloaded from WM. If active maintenance of $S-R$ mappings is necessary, automatic $\mathrm{S}-\mathrm{R}$ translation should not occur when S-R mappings are unloaded. However, if automatic $S-R$ translation depends on memory retrieval, unloading $S-R$ mappings would not help prevent the occurrence of the congruity effect, because memory retrieval is independent of the content of WM.

To test these predictions, we used the task-switching paradigm, for which participants performed two different tasks with different degrees of task uncertainty in the current trial. In that paradigm, RT is shorter when the same task is performed on two consecutive trials (task-repeat trials) than when different tasks are performed (task-switch trials), indicating a task-switching cost (Allport, Styles, \& Hsieh, 1994). The task-switching cost is inversely related to the interval between task cue and target onset (cue-target interval, or CTI), a finding that has been attributed to endogenous preparation of the relevant task set (Meiran, 1996; Rogers \& Monsell, 1995) or to decay of a primed irrelevant task set (Allport et al., 1994). ${ }^{1}$ These accounts suggest that the readiness of relevant task set in WM increases over time, while that of irrelevant task set decreases. Consequently, the active-maintenance account predicts that the response-congruity effect should also decrease with CTI. By contrast, the memory view predicts no influence of CTI on the response-congruity effect, because memory retrieval does not depend on WM.

Meiran (1996) and Rogers and Monsell (1995) found little influence of CTI on the response-congruity effect, inconsistent with the active-maintenance account. However, Meiran's task used spatial stimuli that confounded response congruity with S-R compatibility. Also, Rogers and Monsell used irrelevant stimuli that were congruent, incongruent, or neutral, so two-thirds of the trials in their study were "incongruent," broadly speaking, which might have modulated the congruity effect (Gratton, Coles, \& Donchin, 1992). Therefore, an examination of the influence

\footnotetext{
${ }^{1}$ In addition, see the General Discussion for alternative accounts of the relationship between task-switching cost and CTI (e.g., Altmann \& Gray, 2008; Logan \& Bundesen, 2003; Mayr \& Kliegl, 2000).
}

of CTI on the congruity effect is necessary after excluding these extraneous factors.

At the same time, task uncertainty can also be removed when the task sequence is predictable. Rogers and Monsell (1995) used the alternating-runs procedure, in which participants were informed as to the sequence of two tasks before the trials started. Whereas a reliable taskswitching cost was still obtained with this foreknowledge about the task sequence, it occurred only in the first trial of a series of task-repeat trials after a task switch. RTs for the subsequent task-repeat trials were similar, implying that irrelevant mappings were inhibited or unloaded once the new task had been performed (Schuch \& Koch, 2003). The present study takes a more extreme measure: If irrelevant S-R mappings are unloaded after a switch, unloading should be secured even better if two tasks are separated into two different blocks. Thus, the activemaintenance account would predict no response-congruity effect when the two tasks are blocked, whereas the memory view still predicts a significant congruity effect in that condition.

In the present study, participants performed color and shape discrimination tasks in mixed- or blocked-task conditions. In the mixed-task condition, the two tasks were randomly intermixed and a task cue informed the participants as to the incoming task, and CTI was varied within a block. In the blocked-task condition, participants performed the two tasks in separate blocks that were alternately administered, with a rest period between blocks. Examinations of the responsecongruity effect in these conditions allowed the two accounts of automatic implementation of task-defined mappings to be distinguished.

\section{Experiment 1}

\section{Method}

Participants Forty-eight undergraduate students at Purdue University participated for partial fulfilment of a requirement of their psychology courses. They were randomly assigned to the blocked- or mixed-task condition. All reported having normal or corrected-to-normal visual acuity and normal color vision.

Apparatus and stimuli The apparatus consisted of a personal computer and a 19-in. liquid crystal display color monitor. Participants were seated in front of the monitor at an unrestricted viewing distance of $60 \mathrm{~cm}$. Responses were registered by using a standard keyboard ("z" and "“" keys).

The imperative stimulus was a filled square or circle colored green or red, which appeared within an invisible square (7-cm sides) centered on the screen. The task cue 
was the word COLOR or SHAPE, presented in white against a black background at the screen center, which indicated the forthcoming task. S-R mappings were counterbalanced across participants.

Procedure The experiment was conducted individually in a dimly lit cubicle. Each participant performed eight blocks of 48 test trials. A brief rest period intervened between the blocks. Participants started each block by pressing the space bar.

A trial started with a task cue, which was replaced with a blank display $500 \mathrm{~ms}$ after its onset (see Fig. 1a). The duration of the blank screen varied across trials $(0,500$, and $1,000 \mathrm{~ms}$ ). Thus, the CTI (cue duration + blank) was 500, 1,000 , or $1,500 \mathrm{~ms}$. The target stimulus replaced the task cue and stayed on until a response was made. If the response was incorrect, an error tone occurred $(400 \mathrm{~Hz}$, 500-ms duration). For the blocked-task group, the response deadline was $1,500 \mathrm{~ms}$; if there was no response up to this point, the trial was recorded as "no response," and an error tone was presented. For the mixed-task group, the response deadline was increased to 3,000 ms. This modification was necessary because some participants in a preliminary study

a

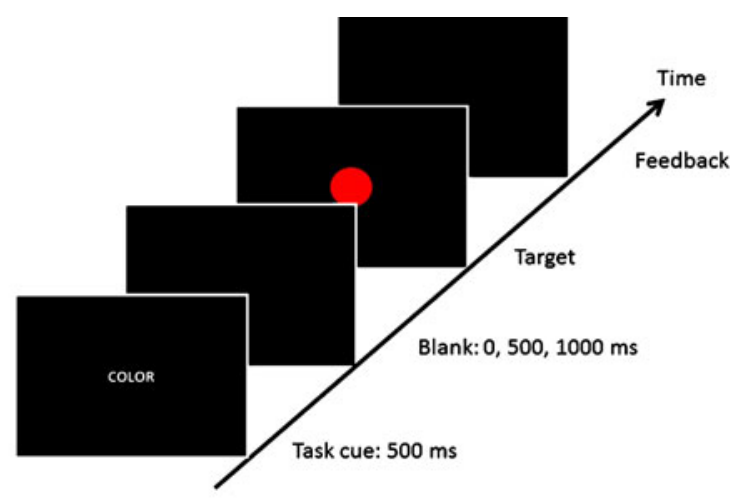

b

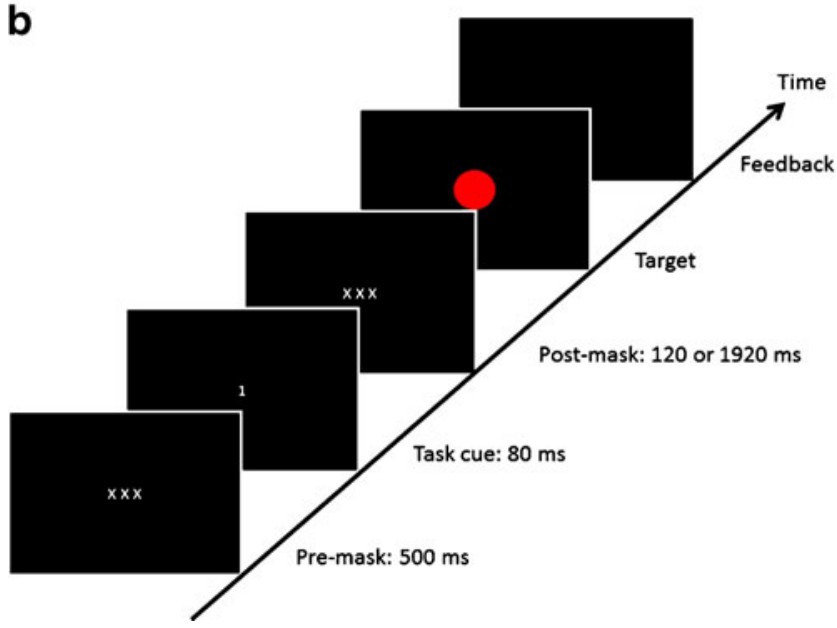

Fig. 1 Schematic illustrations of the trial sequences of a Experiment 1 and $\mathbf{b}$ Experiment 2 had difficulty responding within the 1,500-ms deadline during the practice trials, which resulted in a large error rate. The trial ended with a 1,000-ms blank screen.

For the blocked-task group, each block consisted of a single task, and there were four 48-trial blocks for each task. The color and shape blocks were administered alternately, and the order of these blocks was counterbalanced (ABABABAB or BABABABA). Participants were informed about the relevant task at the beginning of each block, and only one type of task cue appeared throughout the block. Participants were given one block of 24 practice trials for each task prior to the test blocks.

For the mixed-task group, the two tasks were intermixed in a single block, and the relevant task was identified on each trial by a task cue. There were eight 48-trial blocks. Participants performed one 12-trial practice block for each task first, with the order counterbalanced across participants, followed by two 12-trial practice blocks of the mixed condition.

\section{Results}

RTs $<200 \mathrm{~ms}$ were considered anticipations and were excluded from the analysis $(<0.40 \%)$. For the mixed-task group, task sequence (repeat, switch) was determined posthoc after eliminating all trials that immediately followed an error trial and the first trial of each block. Mean RTs for correct responses and percentage errors (PEs) are summarized in Table 1. Our discussions will focus on RTs.

Task unloading The effect of task unloading was analyzed by comparing performance in the blocked-task group and the task-repeat trials of the mixed-task group (for both of which all trials were task repeats) in terms of an ANOVA with CTI, Congruity, and Group as factors (see Table 2).

RT was shorter for the blocked-task group $(M=522 \mathrm{~ms})$ than for the mixed-task group $(M=747 \mathrm{~ms}){ }^{2}$ RT decreased as CTI increased, and this CTI effect was larger for the mixed-task group $(M \mathrm{~s}=800,747$, and $694 \mathrm{~ms}$ for the 500 to $1,500-\mathrm{ms}$ CTIs, respectively) than for the blocked-task group $(M \mathrm{~s}=551,515$, and $500 \mathrm{~ms})$. More importantly, there was a response-congruity effect (see Fig. 2), and the effect was larger for the mixed-task group $(M=60 \mathrm{~ms})$ than for the blocked-task group $(M=15 \mathrm{~ms}){ }^{3}$ Yet, the effect for the

\footnotetext{
${ }^{2}$ Because the response deadlines for the two groups differed, the difference in RTs might have been due to differential speed-accuracy strategies. However, this is unlikely, because PEs for the two groups were equivalent $(2.32 \%$ vs. $2.18 \%$ for the blocked and mixed groups), $F(1,46)<1, \eta_{\mathrm{p}}^{2}=.002$.

${ }^{3}$ To control the overall difference in RTs between the groups, log RT was analyzed in the same manner and showed that this interaction was still significant, $F(1,46)=32.93, M S E=.182, p<.001, \eta_{\mathrm{p}}^{2}=.417$.
} 
Table 1 Mean response time (RT, in milliseconds) and percentage error $(\mathrm{PE})$ as a function of cue-target interval (CTI, in milliseconds) and response congruity for the blocked-task group and for the task-repeat and task-switch trials of the mixed-task group of Experiment 1. Values in parentheses are standard errors of the means

\begin{tabular}{|c|c|c|c|c|c|c|c|c|}
\hline \multirow[t]{2}{*}{ CTI } & \multicolumn{4}{|l|}{ RT } & \multicolumn{4}{|l|}{$\mathrm{PE}$} \\
\hline & \multicolumn{2}{|l|}{ Congruent } & \multicolumn{2}{|c|}{ Incongruent } & \multicolumn{2}{|c|}{ Congruent } & \multicolumn{2}{|c|}{ Incongruent } \\
\hline & Blocked T & ask & & & & & & \\
\hline 500 & 544 & $(12.39)$ & 558 & $(13.73)$ & 1.76 & $(0.02)$ & 3.67 & $(0.02)$ \\
\hline 1,000 & 507 & (12.13) & 525 & $(13.31)$ & 1.31 & $(0.02)$ & 3.27 & $(0.02)$ \\
\hline \multirow[t]{2}{*}{1,500} & 494 & $(14.12)$ & 507 & $(12.91)$ & 1.18 & $(0.03)$ & 2.74 & $(0.02)$ \\
\hline & \multicolumn{8}{|c|}{ Mixed Task: Task-Repeat Trials } \\
\hline 500 & 765 & (31.14) & 835 & $(42.01)$ & 1.07 & $(0.04)$ & 3.25 & $(0.05)$ \\
\hline 1,000 & 724 & $(36.35)$ & 769 & $(41.31)$ & 0.98 & $(0.04)$ & 4.11 & $(0.05)$ \\
\hline \multirow[t]{2}{*}{1,500} & 662 & $(33.81)$ & 726 & $(44.15)$ & 0.51 & $(0.05)$ & 3.18 & $(0.06)$ \\
\hline & \multicolumn{8}{|c|}{ Mixed Task: Task-Switch Trials } \\
\hline 500 & 847 & $(42.38)$ & 954 & $(46.44)$ & 2.50 & $(0.02)$ & 9.24 & $(0.03)$ \\
\hline 1,000 & 774 & $(44.29)$ & 858 & $(44.10)$ & 1.49 & $(0.05)$ & 6.94 & $(0.05)$ \\
\hline 1,500 & 710 & $(41.32)$ & 788 & $(44.87)$ & 1.78 & $(0.04)$ & 7.18 & $(0.03)$ \\
\hline
\end{tabular}

blocked-task group was still significant, $F(1,23)=19.99$, $M S E=402, p<.001, \eta_{\mathrm{p}}^{2}=.465$, consistent with the memory view.

Task-set readiness To test the influence of transient task-set readiness in WM, RTs for the mixed-task group were analyzed in terms of task sequence, congruity, and CTI (see Table 3). RT decreased as CTI increased (850, 781, and $721 \mathrm{~ms}$ for the 500- to 1,500-ms CTIs, respectively). There was a congruity effect (747 vs. $822 \mathrm{~ms}$ for congruent and incongruent) and a task-switching cost (747 vs. $822 \mathrm{~ms}$ for task repeat and task switch). Nevertheless, the congruity effect did not interact with CTI, whereas the interaction between task sequence and CTI was only marginal.

Table 2 ANOVA results for the blocked-task group and task-repeat trials of the mixed-task group of Experiment 1

\begin{tabular}{llll}
\hline Factor & $d f$ & $F$ & $\eta_{\mathrm{p}}^{2}$ \\
\hline CTI & 2 & $58.68^{* * *}$ & .561 \\
CTI $\times$ Group & 2 & $7.48^{* * *}$ & .140 \\
MSE(CTI) & 92 & $(2,538)$ & \\
Cong & 1 & $21.37^{* * *}$ & .317 \\
Cong $\times$ Group & 1 & $7.68^{* *}$ & .143 \\
MSE(Cong) & 46 & $(4,684)$ & \\
CTI $\times$ Cong & 2 & $<1$ & .008 \\
CTI $\times$ Cong x Group & 2 & $<1$ & .017 \\
MES(CTI $\times$ Cong) & 92 & $(2,009)$ & \\
Group & 1 & $34.62^{* * *}$ & .429 \\
MSE(Group) & 46 & $(104,867)$ & \\
\hline
\end{tabular}

$\mathrm{CTI}=$ cue-target interval; Cong $=$ congruity

${ }^{*} p<.05 .^{* *} p<.01 .{ }^{* * *} p<.001$

\section{Discussion}

The results of the experiment are clear in demonstrating that memory retrieval contributes to automatic implementation of task-defined S-R mappings: RT was shorter for the blockedtask group than for the task-repeat trials of the mixed-task group, suggesting that irrelevant S-R mappings were unloaded in the blocked-task group. Yet, the responsecongruity effect was present in the blocked-task condition. Hence, active maintenance of S-R mappings is not necessary for automatic implementation of task-defined S-R mappings.

In contrast, the influence of active maintenance on the congruity effect is not clear: Task-switching cost dissipated

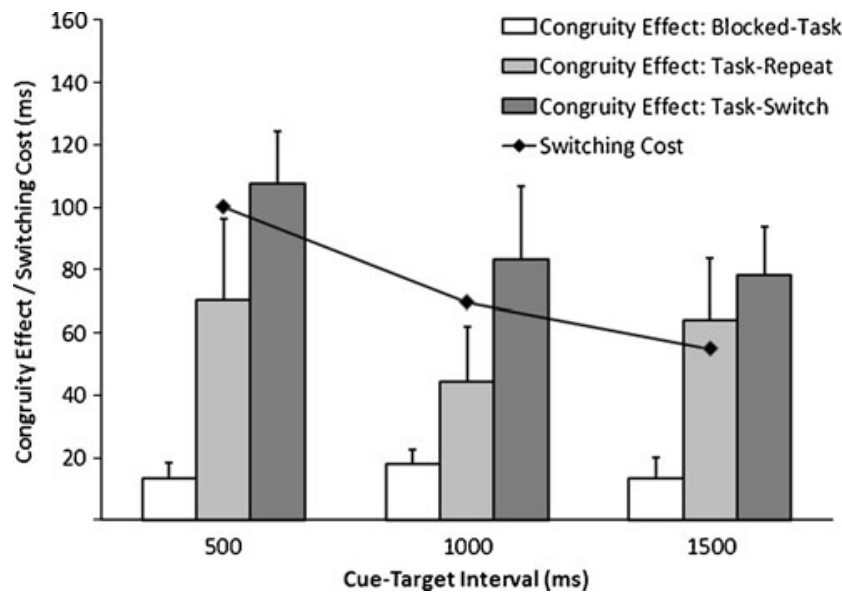

Fig. 2 Response-congruity effects for the blocked-task group and the task-repeat and task-switch trials of the mixed-task group, and taskswitching costs for the mixed-task group, as a function of cue-target interval in Experiment 1 (error bars represent standard errors of the means) 
Table 3 ANOVA results for the mixed-task group of Experiment 1

\begin{tabular}{llll}
\hline Factor & $d f$ & $F$ & $\eta_{\mathrm{p}}^{2}$ \\
\hline Sequence & 1 & $39.47^{* * *}$ & .632 \\
MSE(Sequence) & 23 & $(10,208)$ & \\
CTI & 2 & $41.99^{* * *}$ & .646 \\
MSE(CTI) & 46 & $(9,492)$ & \\
Cong & 1 & $35.89^{* * *}$ & .609 \\
MSE(Cong) & 23 & $(11,178)$ & \\
Sequence $\times$ CTI & 2 & 2.5 & .098 \\
MSE(Sequence $\times$ CTI) & 46 & $(5,129)$ & \\
Sequence $\times$ Cong & 1 & 3.96 & .147 \\
MSE(Sequence $\times$ Cong) & 23 & $(4,098)$ & \\
CTI $\times$ Cong & 2 & 1.12 & .046 \\
MSE(CTI $\times$ Cong) & 46 & $(3,581)$ & \\
Sequence $\times$ CTI $\times$ Cong & 2 & $<1$ & .015 \\
MSE(Sequence $\times$ CTI $\times$ Cong) & 46 & $(3,361)$ & \\
\hline
\end{tabular}

$\mathrm{CTI}=$ cue-target interval; Cong $=$ congruity

${ }^{*} p<.05 .{ }^{* *} p<.01 .{ }^{* * *} p<.001$

(see Fig. 2), but the trend was not statistically significant, which makes it difficult to interpret the lack of interaction between the congruity effect and CTI. This outcome may be due to the insufficiency of the method in inducing endogenous task preparation. Thus, the method was modified in Experiment 2 in four major respects: the use of arbitrary cues (the digits "1" and "2"), a shorter task-cue duration $(80 \mathrm{~ms})$, the use of pre- and postcue masks, and an increased interval between short and long CTIs (200 and $2,000 \mathrm{~ms}$ ). These manipulations have been shown to increase endogenous task preparation (Verbruggen, Liefooghe, Vandierendonck, \& Demanet, 2007).

\section{Experiment 2}

\section{Method}

Participants Twenty undergraduate students were newly recruited from the same subject pool as in Experiment 1, with the same criteria for subject selection.

Apparatus and procedure The apparatus and stimuli were identical with those of Experiment 1, except for the following modifications (see Fig. 1b). A trial started with a precue masking display that consisted of three Xs horizontally arrayed ("XXX") and lasted for $500 \mathrm{~ms}$. The display was replaced by a task cue (the digit "1" or "2") that appeared at the screen center for $80 \mathrm{~ms}$ (in place of the middle $\mathrm{X}$ in the mask), followed by a postcue mask ("XXX") that appeared for 120 or 1,920 ms (CTIs of 200 or
2,000 ms). The target replaced the postcue mask and remained on the screen for $2,000 \mathrm{~ms}$ or until a response was made. An error tone was presented for incorrect responses for $500 \mathrm{~ms}$. As practice, all participants performed one 8-trial block for each task and one 20-trial block for the mixed-task condition, followed by 10 mixed-task blocks of 48 test trials.

Results and discussions

Trials were filtered as in Experiment 1. RT and PE are summarized in Table 4. The ANOVA results are shown in Table 5.

RT was shorter for the 2,000-ms than for the 200-ms CTI (695 vs. $955 \mathrm{~ms}$ ). Both the response-congruity effect (796 vs. $854 \mathrm{~ms}$ ) and the task-switching cost (791 vs. $859 \mathrm{~ms}$ ) were significant, and there was an interaction between congruity and task sequence (see Fig. 3); the congruity effect was larger for task-switch trials than for task-repeat trials ( 75 vs. $41 \mathrm{~ms}$ ). More importantly, task-switching cost dissipated over CTIs (90 vs. $45 \mathrm{~ms}$ ). The responsecongruity effect was smaller for the 2,000-ms than for the 200 -ms CTI (53 vs. $63 \mathrm{~ms}$ ), but only numerically and not statistically.

\section{General discussion}

Distinct mechanisms for response congruity and task switching

The present study investigated the mechanism underlying the automaticity of task-defined mappings, indexed by the response-congruity effect. The memory view of automaticity states that automatic response selection occurs via the retrieval of S-R episodes from LTM. The active-maintenance account argues that automatic $\mathrm{S}-$ $\mathrm{R}$ translation occurs due to erroneous application of $\mathrm{S}-\mathrm{R}$ mappings kept active in WM. In Experiment 1, RT was generally shorter when different tasks were blocked than when they were intermixed in a block. This outcome suggests that irrelevant $\mathrm{S}-\mathrm{R}$ mappings were unloaded from WM when task uncertainty was removed. However, the response-congruity effect was still significant after unloading, suggesting that the effect does not depend on the contents of WM, which supports the memory view but contradicts the active-maintenance account.

Although Experiment 1 failed to show dissipation of taskswitching costs, such dissipation was obtained in Experiment 2 , where several manipulations were implemented to encourage endogenous task preparation (Verbruggen et al., 2007). Yet there was little reduction of the congruity effect over CTIs. Thus, the congruity effect was dissociable from 
Table 4 Mean response time (RT, in milliseconds) and percentage error (PE) as a function of cue-target interval (CTI, in milliseconds) and response congruity for the task-repeat and task-switch trials of Experiment 2. Values in parentheses are standard errors of the means

\begin{tabular}{|c|c|c|c|c|c|c|c|c|}
\hline \multirow[t]{2}{*}{ CTI } & \multicolumn{4}{|l|}{ RT } & \multicolumn{4}{|l|}{$\mathrm{PE}$} \\
\hline & \multicolumn{2}{|l|}{ Congruent } & \multicolumn{2}{|c|}{ Incongruent } & \multicolumn{2}{|c|}{ Congruent } & \multicolumn{2}{|c|}{ Incongruent } \\
\hline & Task-Repe & at Trials & & & & & & \\
\hline 200 & 883 & (27.38) & 930 & $(25.20)$ & 2.10 & $(0.47)$ & 8.80 & (1.11) \\
\hline \multirow[t]{2}{*}{2,000} & 656 & (16.76) & 687 & (24.07) & 1.55 & $(0.59)$ & 3.91 & $(0.72)$ \\
\hline & Task-Switc & ch Trials & & & & & & \\
\hline 200 & 961 & (31.56) & 1035 & (32.13) & 5.42 & $(0.95)$ & 11.17 & $(1.22)$ \\
\hline 2,000 & 679 & (22.72) & 751 & $(25.02)$ & 2.25 & $(0.61)$ & 6.73 & $(0.89)$ \\
\hline
\end{tabular}

task-switching costs, implying that different mechanisms underlie the two phenomena.

Common mechanism for response congruity and task switching

Nonetheless, it should be noted that in Experiment 2 the response-congruity effect also depended on task sequence: The effect was larger for task-switch than for task-repeat trials, which suggests that a common mechanism also underlies the congruity effect and task-switching cost. This partial dissociation between the two phenomena may be understood as implying that multiple mechanisms are responsible for at least one of the two effects.

In the task-switching literature, there has been debate as to the source of dissipation of task-switching costs over CTIs; some assume decreasing irrelevant task-set readiness (Allport e al., 1994), and others assume increasing relevant task-set readiness (Rogers \& Monsell, 1995). These two

Table 5 ANOVA results for Experiment 2

\begin{tabular}{llll}
\hline Factor & $d f$ & $F$ & $\eta_{\mathrm{p}}^{2}$ \\
\hline Sequence & 1 & $47.69^{* * *}$ & .715 \\
MSE(Sequence) & 19 & $(3,828)$ & \\
CTI & 1 & $129.40^{* * *}$ & .872 \\
MSE(CTI) & 19 & $(20,833)$ & \\
Cong & 1 & $18.35^{* * *}$ & .491 \\
MSE(Cong) & 19 & $(7,327)$ & \\
Sequence $\times$ CTI & 1 & $10.11^{* *}$ & .347 \\
MSE(Sequence $\times$ CTI) & 19 & $(2,035)$ & \\
Sequence $\times$ Cong & 1 & $8.08^{* *}$ & .298 \\
MSE(Sequence $\times$ Cong) & 19 & $(1,436)$ & \\
CTI $\times$ Cong & 1 & $<1$ & .021 \\
MSE(CTI $\times$ Cong) & 19 & $(2,245)$ & \\
Sequence $\times$ CTI x Cong & 1 & $<1$ & .003 \\
MSE(Sequence $\times$ CTI $\times$ Cong) & 19 & $(2,356)$ & \\
\hline
\end{tabular}

$\mathrm{CTI}=$ cue-target interval; Cong $=$ congruity

${ }^{*} p<.05 .^{* *} p<.01 .{ }^{* * *} p<.001$ accounts agree with the active-maintenance account under consideration, because they assume that the influence of the irrelevant task set depends on whether it is currently active in WM. Hence, they predict a strong correlation between the two phenomena, which was violated by the results of CTI affecting task-switching cost but not the congruity effect.

The third perspective, the task-cue priming account (Logan \& Bundesen, 2003; Mayr \& Kliegl, 2000), assumes distinct sources for the response-congruity effect and for task-switching costs. For instance, Schneider and Logan's (2005, 2009) priming model attributes dissipation of taskswitching costs to cue encoding and the response-congruity effect to response retrieval, which follows the cue encoding. Consequently, their model predicts no influence of CTI on the congruity effect, consistent with the present results.

In the current form, however, the priming model does not account for the modulation of the response-congruity effect by task sequence. Response retrieval depends on evidence that stimuli provide for particular responses, but the amount of evidence is independent of task sequence. Consequently, the model predicts additive effects of task sequence and response congruity. Thus, a modification is needed in the priming model to fully account for the present results.

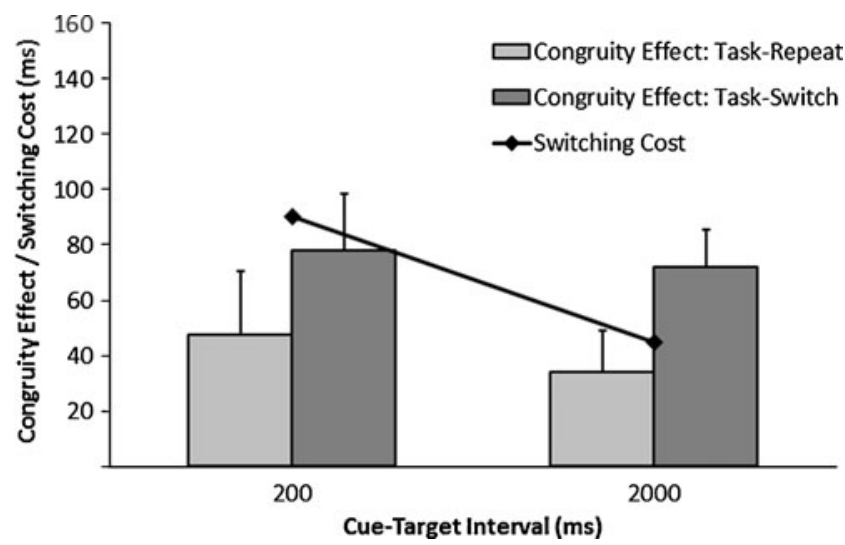

Fig. 3 Response-congruity effects for the task-repeat and task-switch trials of the mixed-task group, with task-switching costs, as a function of cue-target interval in Experiment 2 (error bars represent standard errors of the means) 
A possibility is that the amounts of evidence provided by target and distractor may depend on task sequence, such that evidence from the target is greater and evidence from the distractor is less for task repeats than for task switches, which may result from suppression of LTM representation at response retrieval (Schuch \& Koch, 2003). Alternatively, evidence from the task cue may be varied according to task sequence, such that it is less for task-switch than for task-repeat trials, maybe because the task-set mediator is suppressed (Logan \& Schneider, 2006). In either case, an extension of the priming model would fit the present data better.

\section{Concluding remarks}

The present study suggests that memory retrieval contributes to automatic implementation of task-defined mappings. Throughout the discussion, we have assumed that WM is structurally distinct from LTM (Baddeley, 1992). However, another perspective on WM is also possible. In particular, Cowan's (1988) embedded-process model depicts the memory system as a unitary component in which WM is embedded in rather than separate from LTM. An important feature of the framework is the assumption that WM only delineates a portion of activated LTM (a region of attentional focus), and that some other portions are still active in the background, which make up short-term memory (STM). This model suggests that task-switching costs arise from shifting of attention from the previously attended region (previous task set) to the currently relevant region (current task set). On the other hand, the response-congruity effect can be attributed to STM, which is currently not in the attentional focus but still exerts an influence on performance (see, e.g., Oberauer, 2001).

In a recent study, Meiran and Kessler (2008) offered interpretations of the task-switching performance within the embedded-process framework, and we agree that the framework may provide a parsimonious account of the results from the present study. At the conceptual level, the framework is consistent with Schneider and Logan's (2009) priming model in certain respects (e.g., response selection as memory retrieval), but inconsistent in other respects (e.g., different sources for dissipation of taskswitching costs). It will be interesting to see whether the embedded-process model can also offer a framework for formal models that diverge from existing models in important features.

Author Note M.Y. is now at the Department of Psychology, Vanderbilt University. The research described in the present paper was conducted as a part of the first author's $\mathrm{PhD}$ dissertation research and was supported in part by ARO MURI Grant W911NF-05-1-0153 and the Bilsland Dissertation Fellowship from Purdue University. We thank Gordon Logan, Mei-Ching Lien, Sander Los, and Scott Watter for their insightful comments on earlier drafts. We also thank Giulia
Baroni for her help collecting the data, and Darryl Schneider for discussions on the model.

\section{References}

Allport, D. A., Styles, E. A., \& Hsieh, S. (1994). Shifting intentional set: Exploring the dynamic control of tasks. In C. Umiltà \& M. Moscovitch (Eds.), Attention and performance XV: Conscious and nonconscious information processing (pp. 421-452). Cambridge, MA: MIT Press.

Altmann, E. M., \& Gray, W. D. (2008). An integrated model of cognitive control in task switching. Psychological Review, 115, 602-639.

Baddeley, A. (1992). Working memory. Science, 255, 556-559.

Cohen-Kdoshay, O., \& Meiran, N. (2007). The representation of instructions in working memory leads to autonomous response activation: Evidence from the first trials in the flanker paradigm. Quarterly Journal of Experimental Psychology, 60, 1140-1154.

Cowan, N. (1988). Evolving conceptions of memory storage, selective attention, and their mutual constraints within the human informationprocessing system. Psychological Bulletin, 104, 163-191.

Eriksen, B. A., \& Eriksen, C. W. (1974). Effects of noise letters upon the identification of a target letter in a non-search task. Perception \& Psychophysics, 16, 143-149.

Gratton, G., Coles, M. G. H., \& Donchin, E. (1992). Optimizing the use of information: Strategic control of activation of responses. Journal of Experimental Psychology: General, 121, 480-506.

Hommel, B. (1998). Automatic stimulus-response translation in dualtask performance. Journal of Experimental Psychology: Human Perception and Performance, 24, 1368-1384.

Logan, G. D. (1988). Toward an instance theory of automatization. Psychological Review, 95, 492-527.

Logan, G. D., \& Bundesen, C. (2003). Clever homunculus: Is there an endogenous act of control in the explicit task-cuing procedure? Journal of Experimental Psychology: Human Perception and Performance, 29, 575-599.

Logan, G. D., \& Etherton, J. L. (1994). What is learned in automatization? The role of attention in constructing an instance. Journal of Experimental Psychology: Learning, Memory, and Cognition, 20, 1022-1050.

Logan, G. D., \& Klapp, S. T. (1991). Automatizing alphabet arithmetic: Is extended practice necessary to produce automaticity? Journal of Experimental Psychology: Learning, Memory, and Cognition, 17, 179-195.

Logan, G. D., \& Schneider, D. W. (2006). Interpreting instructional cues in task switching procedures: The role of mediator retrieval. Journal of Experimental Psychology: Learning, Memory, and Cognition, 32, 347-363.

Mayr, U., \& Kliegl, R. (2000). Task-set switching and long-term memory retrieval. Journal of Experimental Psychology: Learning, Memory, and Cognition, 26, 1124-1140.

Meiran, N. (1996). Reconfiguration of processing mode prior to task performance. Journal of Experimental Psychology: Learning, Memory, and Cognition, 22, 1423-1442.

Meiran, N., \& Kessler, Y. (2008). The task rule congruency effect in task switching reflects activated long-term memory. Journal of Experimental Psychology: Human Perception and Performance, $34,137-157$.

Oberauer, K. (2001). Removing irrelevant information from working memory: A cognitive study with the modified Sternberg task. Journal of Experimental Psychology: Learning, Memory, and Cognition, 27, 948-957.

Pashler, H. (1994). Dual-task interference in simple tasks: Data and theory. Psychological Bulletin, 16, 220-244. 
Rogers, R. D., \& Monsell, S. (1995). Costs of a predictable switch between simple cognitive tasks. Journal of Experimental Psychology: General, 124, 207-231.

Schneider, D. W., \& Logan, G. D. (2005). Modeling task switching without switching tasks: A short-term priming account of explicitly cued performance. Journal of Experimental Psychology: General, 134, 343-367.

Schneider, D. W., \& Logan, G. D. (2009). Selecting a response in task switching: Testing a model of compound cue retrieval. Journal of Experimental Psychology: Learning, Memory, and Cognition, 35, 122-136.

Schneider, W., \& Shiffrin, R. M. (1977). Controlled and automatic human information processing: I. Detection, search, and attention. Psychological Review, 84, 1-66.
Schuch, S., \& Koch, I. (2003). The role of response selection for inhibition of task sets. Journal of Experimental Psychology: Human Perception and Performance, 29, 92-105.

Verbruggen, F., Liefooghe, B., Vandierendonck, A., \& Demanet, J. (2007). Short cue presentations encourage advance task preparation: A recipe to diminish the residual switch cost. Journal of Experimental Psychology: Learning, Memory, and Cognition, 33, 342-356.

Waszak, F., Hommel, B., \& Allport, A. (2003). Task-switching and long-term priming: Role of episodic stimulus-task bindings in task-shift costs. Cognitive Psychology, 46, 361-413.

Zbrodoff, N. J. (1999). Effects of counting in alphabet arithmetic: Opportunistic stopping and priming of intermediate steps. Journal of Experimental Psychology: Learning, Memory, and Cognition, 25, 299-317. 